\title{
Long-term efficacy and complications of a multicentre randomised controlled trial comparing Retropubic and Transobturator Mid-Urethral Slings: a prospective observational study.
}

\author{
Ifeoma Offiah ${ }^{1}$ and Robert Freeman ${ }^{2}$ \\ ${ }^{1}$ Affiliation not available \\ ${ }^{2}$ Plymouth Hospitals NHS Trust
}

October 27, 2020

\begin{abstract}
Objective: Concerns exist regarding the risks of mid-urethral slings (MUS) for stress urinary incontinence (SUI), particularly the lack of long-term data. We compare patient reported outcomes of a multicentre randomised controlled trial of retropubic (TVT-GYNECARE) versus transobturator (TOT-MONARC) tape surgery at 12 years. Design and setting: A multicentre study was performed in 11 tertiary referral centres. Population: The 180 participants from the original trial. Methods: Postal questionnaire survey of Patient Reported Outcome Measures using the International Consultation on Incontinence Questionnaire, Patient Global Impression of Improvement questionnaire (PGI-I) and a Numeric rating scale pain questionnaire. Main outcome measures: comparison of the efficacy and complications between the TVT and TOT procedures in the long term. Results: 110/180 responses were received: 55 TVT and 55 TOT participants. Mean follow up: 12.8 years, STD +/- 0.29 years. TVT was significantly superior to TOT in terms of cure ie no SUI: $41.8 \%$ TVT versus $21.8 \%$ TOT ( $\mathrm{p}=0.04$ ). Urgency urinary incontinence (UUI) was the most bothersome urinary symptom: $14.5 \%$ TVT and TOT respondents reported UUI most or all the time. Severe groin/ vaginal pain was reported in $4.8 \%$ TVT and $1.7 \%$ TOT participants. $80 \%$ TVT and $77 \%$ TOT participants reported their symptoms as improved on the PGI-I. Conclusions: TVT is superior to TOT for SUI cure. Efficacy and patient satisfaction are reduced by 12 years. Severe vaginal or groin pain is uncommon. Careful patient counselling of long-term outcomes is required. The MUS appears to be an effective treatment for the majority of women with SUI.
\end{abstract}

\begin{abstract}
Objective : Concerns exist regarding the risks of mid-urethral slings (MUS) for stress urinary incontinence (SUI), particularly the lack of long-term data. We compare patient reported outcomes of a multicentre randomised controlled trial of retropubic (TVT-GYNECARE ${ }^{\mathrm{TM}}$ ) versus transobturator (TOT-MONARC ${ }^{\mathrm{TM}}$ ) tape surgery at 12 years.
\end{abstract}

Design and setting : A multicentre study was performed in 11 tertiary referral centres.

Population: The 180 participants from the original trial.

Methods: Postal questionnaire survey of Patient Reported Outcome Measures using the International Consultation on Incontinence Questionnaire, Patient Global Impression of Improvement questionnaire (PGII) and a Numeric rating scale pain questionnaire.

Main outcome measures: comparison of the efficacy and complications between the TVT and TOT procedures in the long term.

Results : 110/180 responses were received: 55 TVT and 55 TOT participants. Mean follow up: 12.8 years, STD +/- 0.29 years. TVT was significantly superior to TOT in terms of cure ie no SUI: $41.8 \%$ TVT versus 
$21.8 \%$ TOT $(\mathrm{p}=0.04)$. Urgency urinary incontinence (UUI) was the most bothersome urinary symptom: $14.5 \%$ TVT and TOT respondents reported UUI most or all the time. Severe groin or vaginal pain was reported in $4.8 \%$ TVT and $1.7 \%$ TOT participants. $80 \%$ TVT and $77 \%$ TOT participants reported their symptoms as improved on the PGI-I.

Conclusions: TVT is superior to TOT for SUI cure. Efficacy and patient satisfaction are reduced by 12 years. Severe vaginal or groin pain is uncommon. Careful patient counselling of long-term outcomes is required. The MUS appears to be an effective treatment for the majority of women with SUI.

Funding: The United Kingdom Continence Society grant.

Keywords : complications, long-term patient reported outcomes, retropubic mid-urethral sling, stress urinary incontinence, transobturator mid-urethral sling.

Tweetable abstract: The retropubic tape has superior efficacy to the transobturator tape. Complications are comparable and uncommon.

\section{Introduction}

There is international controversy regarding the use of mid-urethral slings (MUS) as evidenced by the July 2020 Baroness Cumberledge inquiry in the United Kingdom which stated that: "little is known about the nature and extent of mesh complications", "every effort should be made to obtain sufficient data" and that "few long-term studies exist" ${ }^{1}$. National and international organisations and a Cochrane review have also called for more long-term data ${ }^{2,3,4,5,6}$.

The MONARC ${ }^{\mathrm{TM}}$ trial, which was a non-inferiority multi-centre randomised controlled trial comparing the retropubic (TVT) and transobturator tape (TOT) procedures was undertaken 12 years ago ${ }^{7}$ and provides an opportunity to address some of the issues above. At 12 months $65.5 \%$ of participants in the TVT group and $63.4 \%$ in the TOT group had no SUI ${ }^{7}$. This follow up study compares the long-term efficacy, patient satisfaction, complications and re-operation rates associated with the MUS in this same patient group using the same patient reported outcome measures (PROM's) as in the original study.

\section{Materials and Methods}

The study was approved by the Health Research Authority and the Research Ethics Committee. REC reference: 17/SW/0110. IRAS project ID: 223334. The study was funded by the United Kingdom Continence Society grant, which was externally peer reviewed for scientific quality: reference SCR/PDK. A follow up postal questionnaire survey of all 180 patients recruited to the original trial was performed ${ }^{7}$. This trial had involved 11 NHS Hospital Trusts and participants were invited by postal survey. A patient information leaflet was provided, and a written informed consent included. All patients who wished to participate in the study were given the option to either return the completed questionnaires in the provided envelopes or request further information if desired. Only one reminder was permitted. Questionnaires were not sent to those who were deceased.

\section{Intervention}

Patients were given 2 questionnaires to complete as in the original study: the validated Patient Global Impression of Improvement (PGI-I) ${ }^{8}$ and the International Consultation on Incontinence Modular Questionnaire Female Urinary Tract Symptoms (ICIQ FLUTS) ${ }^{9}$. A non-validated pain numeric rating scale questionnaire with space for free text comments (Appendices S1) was also used. This questionnaire was added to capture the impact of groin and/ or vaginal pain associated with the mid-urethral slings in the long term. Hospital records of all consented patients were reviewed for complete data. Permission was not given to access those patients notes who had not consented to this follow-up study due to UK research governance regulations.

The primary outcome (as in the original study) was the percentage of participants in each treatment arm who were cured of SUI in the long-term. "Cured" is defined as the answer "no" to question 8a on the ICIQFLUTS 'does urine leak when you are physically active, exert yourself, cough or sneeze?'. The secondary 
objectives included the assessment of satisfaction with the surgery according to the PGI-I, patient reported complications between the two groups as well as persisting or de-novo symptoms such as pain, voiding dysfunction, overactive bladder symptoms or recurrent urinary tract infection. A review of hospital records was performed to establish any further complications and/or the need for further surgery.

\section{Statistical analysis}

Analyses of the main outcomes, comparisons between TVT and TOT, are presented as percent differences with $95 \%$ confidence intervals (CIs) and Fisher's exact test (comparing between groups). Changes over time are presented as percentage change with 95\% CIs and McNemar's test (comparing paired values).

\section{Results:}

Of the 180 participants of the original trial, there were 8 deceased. Invitations were sent to the remaining 172 patients to participate in the follow-up study. 132 responses were received, of which 11 declined to participate. Of the remaining 121 returned questionnaires, 11 were excluded from paired analysis for 1 year versus 12 years due to missing 1 year follow up data. There were consequently 110 participants for paired analysis of endpoint i.e. $61 \%$ of the original trial. No response was received from 40 patients. There was an equal number of retropubic and transobturator tape procedures performed in each group: 55 TVT and 55 TOT participants. Mean follow up was 12.8 years (Range $=12.2$ years to 13.4 years, STD +/- 0.29 years).

\section{Primary outcome :}

\section{Cure of Stress Urinary Incontinence: ICIQ FLUTS}

In the TVT group 23 participants (41.8\%) reported no SUI at 12 years compared with 41 participants $(74.6 \%)$ at 1 year. The difference between periods was significant $32.8 \%$ (95\% CI -49.0 to $14.5 \% \mathrm{p}<0.001)$. In the TOT group 12 participants (21.8\%) reported no SUI at 12 years compared with 38 participants $(69.1 \%)$ at 1 year. The difference between periods was also significant $45.7 \%$ (95\% CI -62.2 to $-30.3 \%$, p < 0.001 ) (Table 1). There was a significant difference between the TVT and TOT groups in cure of SUI at 12 years in favour of TVT $(\mathrm{p}=0.045)$. For the remainder of the participants who reported SUI at 12 years i.e. 32 and 43 for TVT and TOT participants respectively, 20(36.5\%) and 20(36.5\%) had SUI 'occasionally', 5(10\%) and $11(19.3 \%)$ had SUI 'sometimes', $5(8.3 \%)$ and $8(14 \%)$ had SUI 'most of the time', and $2(3.3 \%)$ and $4(8 \%)$ had SUI 'all of the time'.

\section{Secondary outcomes}

\section{Overactive bladder symptoms: ICIQ-FLUTS}

To investigate the effect of surgery on overactive bladder symptoms at 12 years, symptoms were reviewed using questions 3, 4 and 5 on the ICIQ FLUTS. Nocturia and urinary urgency were the most frequently reported symptoms. At 12 years, 24(43.6\%) TVT and 27(49.1\%) TOT participants reported nocturia and $46(83.6 \%)$ TVT and $42(76.4 \%)$ TOT reported any degree of urgency. There are no significant differences between the groups. Despite the high incidences, the majority of patients did not report significant bother: median urinary urgency bother (question 4b ICIQ FLUTS) for the TVT participants was 2 out of 10, and 4 out of 10 for the TOT participants (this difference was not significant).

Urgency urinary incontinence (UUI) was on average the most bothersome urinary symptom at 12 years on the ICIQ FLUTS (question 5b), with a median bother of 3 out of 10 for TVT respondents and 4 out of 10 for TOT respondents. In response to the ICIQ question 5a 'does urine leak before you can get to the toilet', the responses "never" and "occasionally" were analysed as a measure of non-bothersome UUI: 34(61.8\%) TVT and 30(54.6\%) TOT participants had non-bothersome UUI. Of the remaining TVT participants, 13(23.6\%) reported UUI "sometimes", and 8(14.6\%) "most of the time", while 17(30.9\%) of the TOT participants reported UUI "sometimes", 4(7.3\%) "most of the time" and 4(7.3\%) "all of the time". There was no difference in the prevalence of UUI between the TVT and TOT groups at 12 years (p-value 0.56). Overall there was a reduction in UUI prevalence following the procedure: 39 out of 76 participants who had UUI pre-op had 
resolution of UUI at 12 years:(21 TVT and 18 TOT participants). Five TVT and 4 TOT participants had de-novo UUI at 12 years (Figure 1).

Vaginal and groin pain: pain questionnaire

As a paired short versus long term analysis for groin and vaginal pain was not performed, all 121 returned questionnaires were analysed: $63 \mathrm{TVT}$ and $58 \mathrm{TOT}$. The presence of groin and vaginal pain was assessed using the numeric rating scale pain questionnaire. The majority of respondents reported no groin or vaginal pain at 12 years follow up: $52(82.5 \%)$ of the TVT group and $47(81 \%)$ of the TOT group reported no groin pain, while $55(87.3 \%)$ of the TVT group and $50(86.2 \%)$ of the TOT group reported no vaginal pain. Severe groin pain was reported by $3(4.8 \%)$ of the TVT and $1(1.7 \%)$ of the TOT participants, while severe vaginal pain was reported by $3(4.8 \%)$ of the TVT and $1(1.7 \%)$ of the TOT participants (Table 2). One patient reported both having severe vaginal and groin pain, thus the total number of patients with severe pain was $7(5.8 \%)$ of the 121 study participants. Only one of these participants reporting severe pain had a vaginal tape exposure requiring further surgical intervention. Of the remaining 6 participants, 2 had urinary urgency, one had recurrent urinary tract infections causing pain, and the other 3 had no further follow-up after the one-year review. The remainder of participants reported either mild or moderate pain at 12 years. Of note, there were no reports of ' $10 / 10$ ' pain severity on the pain scores. There was no difference between the groups in terms of pain severity or incidence of pain.

\section{c. Patient reported complications}

On the free text space provided for additional comments on the pain questionnaire, $60.9 \%$ of TVT and $71.9 \%$ of TOT participants reported no other problems or concerns related to the surgery at 12 years follow up. Of the remining participants, the most commonly reported complication in both groups was recurrent urinary tract infections: $17 / 62(27.4 \%)$ TVT and 11/58 (19\%) TOT at 12 years. How many were bacteriologically proven is unknown. Urinary retention was the least reported complication, with only 4 TVT (6.5\%) and 2 TOT (3.4\%) participants reporting urinary retention. Of these, 3 TVT and 1 TOT participants required intermittent self-catheterisation and the other 2 resolved spontaneously. There was no statistical difference between the groups.

Analysis of the additional comments offered by the patients revealed that the majority of women were pleased with their procedure. Forty-three participants provided free text comments: 19 were positive comments from participants who were satisfied with their procedure and had no complications, and 24 participants provided negative comments.

Satisfied comments included "the operation transformed my life enabling (me) to do all the sports I love without worrying", "I am extremely happy with the outcome of this operation", "it has changed my life for the better", " thank you very much".

Five of the 19 women who were initially happy with the procedure and reported no complications, had observed a deterioration in efficacy of surgery after approximately 8-10 years: "I was very pleased with the initial result", "improved things for a while", "for the first 8-9 years it was excellent"; "until approximately 2 years ago it was brilliant".

The negative comments associated with the procedures were similar to those reported in the Cumberledge inquiry e.g. chronic vaginal pain precluding sexual intercourse, overactive bladder symptoms and urinary hesitancy. These comments included: "intercourse can be a little painful", "sexual intercourse ceased following surgery because of severe pain", "husband a little frustrated by this situation". Negative comments associated with bladder symptoms include: "I have to go to the toilet as soon as I realise", "I wet the bed", "very irritable bladder", "bladder not emptying properly", "I have to catheterise 4 times daily".

Satisfaction with the surgery: PGI-I All 121 returned PGI-I questionnaires were assessed as PGI-I is not validated for comparison of change over time. Analysis revealed 51(79.7\%) TVT and 44(77.2\%) TOT participants reported their symptoms were either "very much better", "much better" or "a little better" at 12 years. The difference in satisfaction between the TVT and TOT groups at 12 years is not significant (p 
$>0.05)$ (Table 3). One (1.6\%) participant in the TVT group and 9 (15.8\%) from the TOT group reported no change in their symptoms at long term follow up. The remainder reported a dissatisfaction with the procedure, reporting their symptoms as either "a little worse", "much worse" or "very much worse" at 12 years: $12(18.8 \%)$ of the TVT and $4(7 \%)$ of the TOT groups. There was no statistical difference between the groups.

Hospital records review

The hospital records of consented patients only (in keeping with UK research governance regulations) were reviewed for additional information. They were unavailable for 8 patients, therefore the 12 year outcomes for 113 participants who consented to the follow up study were reviewed. Analysis revealed 86 participants who required no further urogynaecology input following the 1year assessment. Of the remaining 27 participants who returned to urogynaecology clinics following the primary surgery, the most common presenting complaint was overactive bladder symptoms: 13/27 (48\%):7 TVT and 6 TOT participants. They were managed with bladder retraining, anticholinergics or intravesical botulinum toxin injections. Fifteen participants had recurrent urinary tract infections (UTI's). These were managed in the community by the General Practitioners. Only 8 of the 15 with recurrent UTI's were seen in secondary care.

Vaginal and/or groin pain was noted in 6 participants. Only one of these had reported severe pain on the pain questionnaire. This participant had vaginal tape exposure post-TOT procedure, which was excised. She was subsequently diagnosed with bladder transitional cell carcinoma (TCC). She had no bladder tape extrusion (i.e. into the bladder), and the TCC was associated with a clear cell carcinoma of the right kidney, for which she required a laparoscopic nephrectomy.

The remaining 5 participants identified from the hospital record review with pain, reported either mild or moderate pain on the pain questionnaire. This was associated with vaginal tape exposure in 3 participants (2.5\% of study population: all TVT procedures) and required return to theatre for tape excision. Following this, resolution of pain was noted in 2 participants. The other participant is currently undergoing treatment for chronic groin, lumbar and hip pain. The final 2 patients with pain not related to tape exposure, had recurrent UTIs which resolved following low dose antibiotic treatment and eradication of the UTI.

Six patients (2 TVT and 4 TOT) had ongoing stress urinary incontinence requiring further surgery. These were managed with repeat retropubic tape procedures, Bulkamid urethral injections, autologous facial slings or a combination of these.

Voiding dysfunction requiring intermittent self-catheterisation was present in 4 participants (3 TVT, 1 TOT). One participant had no complications or problems associated with the mesh but presented for review due to anxiety following the media reports about transvaginal mesh.

\section{Discussion:}

Main findings:

The Cumberledge enquiry has highlighted a lack of long-term outcomes for the mid-urethral tape procedures $(\mathrm{MUS})^{1}$. As recommended in this inquiry, as well as the ICI guidelines ${ }^{10}$, patient perception of outcomes using patient reported outcome measures (PROM's) were used to evaluate long terms outcomes 12 years after TVT and TOT procedures. PROMS's used in this follow-up as well as the original study, reduces the bias of health care personnel's impression of outcome. The 12year results show that TVT is superior to TOT for cure of stress urinary incontinence in the long-term and that the two procedures are comparable in terms of complications. However, there was a decline in success and satisfaction with time, as seen with other surgeries for SUI such as colposuspension.

For the assessment of success, the primary study defined 'cure' as 'never' on the ICIQ FLUTS questionnaire Q8a: "does urine leak when you are physically active, exert yourself, cough or sneeze?"7 . The same was used in this long-term follow-up study. This might be regarded as a strict definition. For example, there was a large proportion of women in this study who reported SUI 'occasionally' only i.e. $35 \%$ TVT and $36 \%$ TOT. 
This level of incontinence was not regarded as bothersome. Despite the lower subjective success rate, both the TVT and TOT produced significant improvement in symptoms according to the PGI-I for the majority of participants.

Objective and subjective cure rates for the MUS differ depending on the length of follow up, criteria used for definition of cure and the instruments used for assessing cure e.g. questionnaires, pad tests or urodynamic findings. The long term ( $>5$ years) efficacy and safety of the MUS procedures are summarised in a systematic review by Maggiore and colleagues ${ }^{11}$. They report an objective cure rate for the TVT and TOT procedures of $57.2 \%$ and $68.8 \%$ respectively ${ }^{11}$. Nilsson and colleagues reported a $90 \%$ objective continence rate following retropubic tape procedure based on cough test, and $87 \%$ continence rate based on PGI-I at 17 years follow$\mathrm{up}^{12}$. Since then further reports on long-term efficacy on all types of MUS have been published with cure of SUI ranging from $65 \%$ to $97 \%^{13,14,15,16}$.

Interpretation:

These differences in outcomes can also be attributed to the patient population, with studies with higher cure rates excluding patients with mixed incontinence. Our study includes patients with SUI-predominant mixed incontinence. It is therefore recommended that all studies use standardised outcome measures which will enable systematic reviews and meta-analyses to inform clinical practice and patient counselling e.g. as recommended by $\mathrm{ICI}^{10}$.

The mid-urethral sling procedure was placed under high vigilance restriction by the FDA in 2011 and the UK NHS Improvement (NHS England) in 2018. This was secondary to reports of pain and complications in some women following tape insertion and highlighted in the Cumberledge report ${ }^{1}$. This led to public campaigns and legal action to raise awareness along with subsequent media reports ${ }^{17}$. The true extent of the problem is unclear thus this study aimed to assess the proportion of women who reported pain and its severity long-term. The results showed that the majority of women were pain-free. For those who reported moderate pain, it did not have a significant impact on quality of life and surgical intervention was required for a minority. For the $6 \%$ of women with severe pain, their quality of life was diminished. It is therefore important to emphasise the need for appropriate pre-operative counselling about this risk. Early surgical intervention for complication management is recommended to achieve symptom resolution ${ }^{18}$.

An attempt to understand why women have a different response to the same treatment is important. Greater attention to patient selection, preoperative assessment and surgical technique might be critical to optimizing outcomes from the procedures ${ }^{19}$.

Our study respondents are 12 years older and it could be argued that the urinary urgency and UUI reported are a result of ageing rather than the long-term effects of surgery ${ }^{20}$. However, comparison of the baseline prevalence with the 12year follow-up data confirmed a decrease in the number of participants reporting these symptoms. The population recruited to the original trial was comprised of women with mixed incontinence: 93.5\% TVT and 97\% TOT group had reported any UUI prior to their surgery and $87 \%$ TVT and $89 \%$ TOT group reported any urinary urgency ${ }^{7}$. Consequently, though the prevalence of UUI and urinary urgency at 12 years is deemed high, comparison with the baseline prevalence of both symptoms confirms a reduction of these symptoms at 12 years for both the TVT and TOT groups. In addition, urinary urgency and UUI did not represent significant bother for the majority of patients.

\section{Strengths:}

A number of studies exist, which analyse the long-term outcomes of MUS. This study represents one of few studies which specifically compares the efficacy and complications between the TVT and TOT procedures in the long term. The study thus provides unique information on the superior efficacy of the TVT procedure in direct comparison to the TOT procedure in a randomised controlled trial setting.

Additionally, we specifically addressed the pain and complications issue presented by the adverse media reports, by using both a numeric rating scale for pain and a free text space for additional comments. This has the advantage of giving the patients a platform to report any issues they have experienced with the 
tapes. We are confident that the participants report of no complications was accurate, as it is possible that the adverse media reports could result in patient anxiety and self-referral for assessment. Thus, the inclusion of a free text box for added comments represents a strength of the study.

Limitations:

Statistical analysis could not be performed to compare pain scores between the TVT and TOT procedures because the majority of respondents ( $>80 \%$ in both groups) reported no pain. However, the trend for no difference between the groups is apparent.

Additionally, analysis of all returned questionnaires at 12 years was limited as there was incomplete information from 11 participants from the 1year study. Consequently, despite receiving 121 returned questionnaires, paired ICIQ-FLUTS analysis between long- and short-term outcomes could only be performed on 110 participants, reducing the data available for analysis from $70 \%$ to $64 \%$.

Due to the UK research governance regulations, General Practitioner records could not be accessed to identify other issues which were managed in the community. Likewise, the hospital records of those patients who had not given consent for participation in this follow- up trial could not be accessed. This represents the main limitation of the study. The response rate of $70 \%$ could have been improved if further reminders had been sent, but only one was allowed by research ethics.

\section{Conclusion:}

This study was performed to identify long-term data on the efficacy and complications associated with the mid-urethral tape procedures. The results suggest that the retropubic procedure is superior to the transobturator procedure in terms of cure for SUI. Continence declines significantly over time for both procedures, but they are comparable in terms of low rates of complications, with the majority of patients being satisfied with the outcome. This lends further support to the view that MUS's, particularly the retropubic tapes, are an effective long-term treatment option for the majority of women with SUI provided detailed information and counselling are provided.

\section{Acknowledgements:}

This work was funded by a grant from the United Kingdom Continence Society.

Disclosure of Interests: the authors have no conflicts of interest to disclose.

\section{Contribution to Authorship:}

IO : Protocol/project development, Data collection, management and analysis, Manuscript writing and editing, final approval of the paper

RF : Study lead, protocol/project development, Data analysis, Manuscript editing and approval of the final draft

MW : Data and statistical analysis, editing of the paper, final approval of the paper

CM : Data collection, final approval of the paper

AS : Data collection, final approval of the paper

MJ : Data collection, final approval of the paper

TH : Data collection, final approval of the paper

SF : Data collection, final approval of the paper

KL: Data collection, final approval of the paper

SB : Data collection, final approval of the paper

MS : Data collection, final approval of the paper 
NC : Data collection, final approval of the paper

FR : Data collection, final approval of the paper

PA: Original study concept and review of final draft.

\section{Details of Ethics Approvals:}

The study was approved by the Health Research Authority and the Research Ethics Committee. Approval date 19 December 2017. South West - Cornwall and Plymouth.

REC reference: 17/SW/0110. IRAS project ID: 223334.

Funding: This work was funded by a grant from the United Kingdom Continence Society.

\section{References:}

1. https://www.immdsreview.org.uk/downloads/IMMDSReview_Web.pdf

2. NICE (2003) Final Appraisal Determination Tension-free vaginal tape (Gynecare TVT) for stress incontinence. National Institute for Health and Clinical Excellence, January 2003.

3. Smith A, Bevan D, Douglas HR, James D. Management of urinary incontinence in women: summary of updated NICE guidance. BMJ, 2013. 347: p. f5170.

4. The Scottish Independent Review of the Use, Safety and Efficacy of Transvaginal Mesh Implants in the Treatment of Stress Urinary Incontinence and Pelvic Organ Prolapse in Women. Interim Report. October 2015

5. SCENIHR Opinion on the safety of surgical meshes used in urogynecological surgery. December 2015.

6. Ford AA, Rogerson L, Cody JD, Aluko P, Ogah JA. Mid-urethral sling operations for stress urinary incontinence in women. Cochrane Database of Systematic Reviews 2017, Issue 7 . Art. No.:CD006375.DOI:10.1002/14651858.CD006375.pub4

7. Freeman R, Holmes D, Hillard T, Smith P, James M, Sultan A, et al. What patients think: patient reported outcomes of retropubic versus trans-obturator mid-urethral slings for urodynamic stress incontinence. A multicentre randomised controlled trial. Int Urogynecol J. 2011 Mar;23(3):279-86.

8. Yalcin I, Bump RC. Validation of two Global Impression questionnaires for incontinence. Am J Obstet Gynecol. 2003 Jul;189(1):98-101.

9. Homma Y, Yoshida M, Yamanishi T, Gotoh M. Core Lower Urinary Tract Symptom Score (CLSS) questionnaire: a reliable tool in the overall assessment of lower urinary tract symptoms. Int J Urol. 2008 Sept;15(9):816-820.

10. Abrams P, Andersson KE, Apostolidis A, Birder L, Bliss D, Brubaker L, et al. $6^{\text {th }}$ International consultation on Incontinence. Recommendations of the International Scientific Committee: Evaluation and treatments of urinary incontinence, pelvic organ prolapse and faecal incontinence. Neurourol Urodyn. 2018 Aug;2(37):2271-2272.

11. Maggiore ULR, Agri EF, Soligo M, Marzi VL, Digesu A, Serati M. Long-term outcomes of TOT and TVT procedures for the treatment of female stress urinary incontinence: a systematic review and meta-analysis. Int Urogynecol J. Aug 2017;28(8):1119-1130.

12. Nilsson CG, Palva K, Aarnio R, Morcos E, Falconer C. Seventeen years' follow-up of the tension-free vaginal tape procedure for female stress urinary incontinence. Int Urogynecol J, 2013. 24(8): p. 1265-9.

13. Bakas P, Papadakis E, Karachalios C, Liapis I, Panagopoulos N, Liapis A. Assessment of the long-term outcomes of TVT procedure for stress urinary incontinence in a female population: results at 17 years follow-up. Int Urogynecol j. 2019 Feb;30(2):265-269.

14. Bakas P, Papadakis E, Karachalios C, Beta A, Liapis A. Long-term efficacy follow-up of tension free vaginal tape obturator in patients with stress urinary incontinence with or without cystocoele. Int $\mathrm{J}$ Gynecol Obstet. Dec 2018;143(3):339-343.

15. Serati M, Braga A, Athanasiou S, Tommaselli GA, Caccia G, Torella M, et al. Tension-free Vaginal Tape-Obturator for Treatment of Pure Urodynamic Stress Urinary Incontinence: Efficacy and Adverse Effects at 10-year follow-up. Eur Urol. 2017 Apr;71(4):674-679. Doi 10.1016/j.eururo.2016.08.054. 
Epub 2016 Sep 3.

16. Abdel-Fattah M, Cao G, Mostafa A. Long-term outcomes for transobturator tension-free vaginal tapes in women with urodynamic mixed urinary incontinence. Neurourol Urodyn. 2017 Apr;36(4):902-908.

17. Karmakar D, Hayward L. What can we learn from the vaginal mesh story? Climacteric. 2019 Jun;22(3):277-282.

18. Offiah I, Rachaneni S, Dua A. Management of mid-urethral tape complications: a retrospective study. J Obstet Gynecol India. 2019 Sept. 70(2):152-157. doi: 10.1007/s13224-019-01269-5.

19. Toozs-Hobson P, Cardozo L, Hillard T. Managing pain after mesh implants in pelvic surgery. Eur J Obs Gyn Rep Biol. 2019;234:49-52.

20. Ranson RN, Saffery MJ. Neurogenic mechanisms in bladder and bowel ageing. Biogerontology. 2015 Apr;16(2):265-84.

\section{Tables}

Table 1

\section{Hosted file}

image1.emf available at https://authorea.com/users/370544/articles/489160-long-termefficacy-and-complications-of-a-multicentre-randomised-controlled-trial-comparingretropubic-and-transobturator-mid-urethral-slings-a-prospective-observational-study

Table 1: ICIQ FLUTS Q8a. Contingency Analysis of any Stress Urinary Incontinence (SUI) at 12 years by SUI at 1 year. Retropubic tape (TVT) and Transobturator (TOT) procedures significant, $\mathrm{p}<0.001$. Statistical analysis: 1 year versus 12 years using McNemar's test. TVT versus TOT analysis at 12 years using Fisher's exact test.

Table 2

\section{Hosted file}

image2.emf available at https://authorea.com/users/370544/articles/489160-long-termefficacy-and-complications-of-a-multicentre-randomised-controlled-trial-comparingretropubic-and-transobturator-mid-urethral-slings-a-prospective-observational-study

Table 2: Subjective assessment of groin and vaginal pain scores. there was no difference between the two groups in relation to groin or vaginal pain. The majority of participants had no pain at twelve years follow up. Indicated is participant number and percentages. Total TVT $=63$, Total TOT $=58$.

Table 3

\section{Hosted file}

image3.emf available at https://authorea.com/users/370544/articles/489160-long-termefficacy-and-complications-of-a-multicentre-randomised-controlled-trial-comparingretropubic-and-transobturator-mid-urethral-slings-a-prospective-observational-study

Table 3: Patient Global Impression of Improvement at 12 years. There was a trend towards increased satisfaction by the TVT participants, however this was not significant. Statistical test Fisher's exact test.

\section{Hosted file}

Figures BJOG.pdf available at https://authorea.com/users/370544/articles/489160-1ongterm-efficacy-and-complications-of-a-multicentre-randomised-controlled-trial-comparingretropubic-and-transobturator-mid-urethral-slings-a-prospective-observational-study 